\title{
Research on Causes and Prevention of Incision Infection in Patients Undergoing Abdominal Surgery in Department of Gynecology Xiuming Chen ${ }^{1, a}$ \\ ${ }^{1}$ Baotou City Gynaecology Hospital, Baotou, 014010, China
}

Keywords: Department of gynecology, Incision infection, Abdominal surgery

\begin{abstract}
Objective: To explore the risk factors of incision infection in abdominal surgery in the department of gynecology. Methods: A total of 240 patients who underwent abdominal surgery in Baotou City Gynaecology Hospital were enrolled in the study, including 120 incision infection patients and 120 non- infection patients to explore the risk factors of wound infection and to discuss the corresponding prevention countermeasures. Results: After comparison, we can find that the proportion of incision infection caused by obesity is the highest. Other causes of infection in gynecology abdominal surgery include too long operation time, patients with obesity, no preventative medicine, polluted clinical ward, delayed dressing and low disinfection. All the 120 patients were healed by reasonable treatment. Conclusion: We should take the corresponding preventive measures to improve the quality of operation.
\end{abstract}

\section{Introduction}

Surgery plays an important role in the treatment of gynecological diseases, but surgical treatment is the process of trauma. Combined incision infection can increase the pain of patients, prolong the length of stay, and affect the quality of life. In this study, we retrospectively analyzed the clinical data of patients in Baotou Gynaecology Hospital. We analyzed the risk factors of incision infection and discussed the preventive measures of nursing care in patient.

\section{Data and Methods}

Data. From June 2013 to June 2016, 120 patients with abdominal incision infection after gynecological surgery in our hospital were selected as the clinical observation, the average age of the patients was from 22 to 74 years old. In addition, 120 cases of patients with incision infection after abdominal surgery were selected as control group, the age span was 23 40 years, with an average age of 32.4 years. There was no significant difference between the two groups in terms of operation type, age, history and so on.

Infectious Standards. Abdominal incision infection after gynecologic surgery. Non-incisional infection: incision liquefaction, incision suture with a small amount of secretions, accompanied by mild inflammation. Superficial infection of incision: the skin and subcutaneous tissue involved in incision, infection in 1 months, superficial secretion in the superficial incision and pain, swelling and heat. Or red mouth incision deep infection symptoms) and organ infection refers to the incision in deep soft tissue or other anatomical sites outside of incision infection, purulent incision, patients with local pain or fever; drainage of pus can deep wound of soft tissue or other organs, and the bacterial culture results show positive. Confirmed by histopathology or B ultrasound or X-ray examination, the diagnosis of deep infection. Surgical incision infection was divided into three grades, namely deep incision infection, superficial incision infection and non-incisional infection. Deep incision infection refers to the site of infection is the deep incision of the soft tissue and the incision outside the anatomical site, the patient will appear in these parts of the pus, the incision inside the purulent secretions. According to the above criteria, 120 patients were non-incisional infection, and the other 80 patients had superficial infection of incision, and the other 24 patients had deep incision and organ infection.

Methods.The clinical data of the two groups were compared and analyzed, and the risk factors of incision infection were analyzed. The collected data were processed by SPSS19.0 software. 


\section{Results}

By contrast can be found, the patients in the observation group due to wound infection caused by obesity accounted for $32.5 \%$, because the operation caused by long time the proportion is $22.5 \%$, the disinfection is not in place due to the proportion of $12.5 \%$, due to diseases caused by not prophylaxis ratio of $16.7 \%$, due to pollution caused by ward for $9.2 \%$ of the proportion of the delay caused by dressing ratio was $6.7 \%$. All the 120 patients were healed by reasonable treatment. The Infected factors of the observation group is show in the following table.

Table 1. Infected factors of the observation group

\begin{tabular}{|c|c|c|}
\hline Infected factor & Number of cases & Rate \\
\hline Low disinfection & 15 & $12.5 \%$ \\
\hline Too long operation time & 27 & $22.5 \%$ \\
\hline Patients with obesity & 39 & $32.5 \%$ \\
\hline No preventative medicine & 20 & $16.7 \%$ \\
\hline Polluted clinical ward & 11 & $9.2 \%$ \\
\hline Delayed dressing & 8 & $6.7 \%$ \\
\hline
\end{tabular}

\section{Causes of Incision Infection in Undergoing Abdominal Surgery in Department of Gynecology}

For different patients, the probability of wound infection after abdominal surgery is different, that is, in the risk factors of incision infection, the patient's own condition is one of the key factors. These factors included the age of the patient, the grade of the surgery, the level of hemoglobin, the weight and length of hospital stay, and the presence of underlying disease. For example, older patients, or lower hemoglobin levels and with underlying diseases, the immune capacity will be lower than the normal level, relatively easy to cause the infection of incision; moreover, obese patients with incision infection will increase the chance of the reason is that the thick layer of fat can easily lead to invalid cavity formation the surgical site, exposure and operation will have a direct impact, the operation time was forced to extend is a major factor. The operation time, the longer the improper operation type, non-surgeon to suture, will increase the chance of patients with incision infection. This is because the operation time is longer, the greater the probability of bacterial infection, incision infection probability will be higher; technical level of operation personnel will directly determine the incidence of postoperative infection; extended hand personnel surgical suture will cause the operation time, and even increase the amount, resulting in secondary unnecessary infection. Before and after surgery and perioperative use of antibiotics in no patients, incision infection after abdominal surgery opportunity showed significant increasing trend, but due to the patient or hospital, in the preoperative and perioperative period, patients were not in accordance with the provisions of the standard use of antibiotics, and that patients with autoimmune the state is at a relatively low level, postoperative incision infection was closely related with this. In addition to the above risk factors for postoperative infection in the Department of Obstetrics and Gynecology, the following factors have a negative impact on maternal health. For example, because of the premature rupture of membranes, the bacteria present in the lower genital tract may enter the uterine cavity or the amniotic cavity. If at this time is a cesarean section, abdominal incision is likely to create the bacteria into the incision conditions, causing infection. The age, physical decline reduced immunity, the probability of infection is high. Patients with obesity, is a thick subcutaneous fat, poor blood supply, resulting in a decline in the ability of anti-infection, easily due to improper suture in fat left hemocoel, dead cavity caused by infection, and obesity patients prone to fat liquefaction also, prone to infection. The longer the 
operation time, the wound and related medical equipment exposure in the air will be longer, resulting in a large increase in the probability of infection. In the course of the operation, without the use of antibiotics, the probability of infection will increase. Personal care properly or not is the last barrier, proper care, keep the body clean, positive attitude and drink attention. The probability of infection will be reduced.

\section{Prevention of Incision Infection in Undergoing Abdominal Surgery in Department of Gynecology}

Preoperative Preparation.Preoperative evaluation of patients' tolerance and recovery expectations. In order to improve the nutritional status and immune function of elderly patients with severe disease and poor nutritional status. Clinical data show that the operation time is too long, incorrect medication during the operation, it is also easy to cause the incision infection. The hospital itself and medical personnel itself should enhance the sense of responsibility, strengthen the management responsibility and operation standards, improve work skills and proficiency of medical staff, reduce the operation time, reduce the probability of infection of subjective abdominal incision after surgery, improve the relationship between doctors and patients. Reducing the length of stay is an effective way to reduce the incidence of postoperative abdominal infection in normal women. Pelvic encapsulated effusion is pelvic inflammatory exudate, can occur in pelvic inflammation, annex inflammation or endometriosis and various types of abdominal surgery, tissue swelling cells, slightly viscous fluid is around the tissue gradually formed a cystic mass. If not treated, you can slowly grow up. Generally larger package, the drug cannot be eliminated, the need for surgical removal. The occurrence of pelvic inflammation and patients with poor health habits, such as menstruation, postpartum sexual life in January, January after gynecological surgery in the tub. Artificial abortion, induction of labor caused by severe disinfection of iatrogenic infection, can also cause pelvic effusion. Especially in surgical patients with a history of chronic pain, if often have abdominal don't careless, to check as soon as possible, in order to find the disease as soon as possible. In general, the length of stay should be within a week of the best, and in the case of conditions permitting early surgery. This effect will be better, and can significantly reduce the probability of colonization of drug-resistant strains in the skin and mucous membrane of patients. For obese women, the use of lower abdominal arc incision, reduce postoperative suture difficulties.

Intraoperative Implementation.In order to reduce the time of operation and ensure the effect of suture, we should strictly implement the principle of asepsis. Strictly carry out routine operation and technical principles, operation should be done quickly and accurately, reduce tissue damage. Involved in the operation of the division of nursing staff clear, trained, and close cooperation with the surgeon, so that surgery in an orderly manner. Patients with high body mass index as far as possible to shorten the time of contact with the adipose tissue, to avoid repeated cutting tissue and fat tissue bleeding point of the blind electrocoagulation hemostasis. Suture of subcutaneous tissue with saline before the necrosis of adipose tissue rinse. In the early period of dressing change, the incision should be observed, and the proper pressure should be extruded from the incision. On the one hand, the leachate can be extruded to avoid the occurrence of fat liquefaction. On the other hand, the infection can be found early, which is helpful for early treatment. Elderly patients should try to improve the general condition of the patients; active treatment of various diseases, correct the anemia and hypoproteinemia, malnutrition; vaginal examination, anus check not too frequent, carefully chosen according to the actual needs of patients undergoing elective surgery; if the condition allows, try to choose the suitable temperature in the season; fully clean skin incision, the shortest possible time of skin preparation before operation, so as not to cause two pollution; obesity and pay attention to the protection of fat, with high frequency electric knife; suturing peritoneum with lots of physiological saline rinse off the incision, adipose tissue; a proper interval suture, suture moderate tightness, if necessary, reduce suture; improving the surgeon professional technical proficiency and technical level, to strengthen the supervision and guidance of the young doctor suture technique; intraoperative surgical staff involved in the clear division of labor, to cooperate, to Shorten operation time. Surgery 
should be performed if possible. Intravenous infusion of prophylactic antibiotics before anesthesia can effectively reduce the incision infection and shorten the length of hospital stay.

Postoperative Nursing.Doctors in the distribution of drug resistance and rational use of antimicrobial agents, and should control the use of antimicrobial drugs. At the same time, the doctor should carry out a comprehensive rehabilitation education to maintain a good state of mind, which is helpful to the recovery of the patient's resistance and wound healing. In the process of nursing care is not clean, bad attitude, lack of nutrition, but also increase the incidence of infection. The nurses in the nursing process, positive guidance on the mentality of the patients, in order to improve the immunity of patients, and to improve the cleanliness of the body, controlling the sources of pollution, strengthen nutrition, promote wound healing, improve the ability of anti-infection, reduce the infection probability. Even because of various reasons of postoperative abdominal incision infection has occurred, should also care to the daily observation on infection status and recovery status, keep clean, rehabilitation education of patients, reduce the psychological pressure of the patients. To strengthen drinking contributes to wound healing and improve the anti-infection ability, fasting spicy spicy. To overcome the pain from the hands as soon as possible to improve their own immunity, cure the infection. Infected by the infected wound care. Abdominal incision is generally third days after the change of dressing and check whether there is any wound or tenderness. There is skin suture, generally seventh days after the removal of stitches. Vaginal hysterectomy for patients with vaginal bleeding, vaginal bleeding should be observed, pay attention to whether there are vaginal secretions and their color, character, quantity, whether or not. Postoperative care is the care of patients from the end of surgery to the hospital before discharge. The purpose of postoperative care is to reduce the discomfort and pain of patients, prevent postoperative complications, and promote early rehabilitation of patients. Patients and their families should be actively involved in nursing activities, nurses in the care of patients. At the same time, nurses help patients and their families to learn how to deal with their own strengths.

\section{References}

[1] Luo Caizhen. Influencingfactorsforabdominal surgeryincisioninfections indepartmentof obstetricsandgynecologyandpreventioncountermeasures [J].Chinese Journal of Nosocomiology, 2013, 23(2): 368-370.

[2] Zou Lijuan. TheCausesandNursingofAhdominalIncisionInfectionafter Operation inDepartmentofGynecologyandobstetrics[J]. China Continuing Medical Education, 2015, 7(16): 197-198.

[3] RuanXiaocui, Zhou Maoying, Zhang Tengfei, Analysis of Risk Factors and Preventive Measures of Abdominal Incisionin Obstetric and Gynecological Infections[J]. Chinese Journal of Nosocomiology, 2010, 20(7): 935-936.

[4] Xu Qiuhong, Risk Factors Analysis and Prevention of Incision Infection after AbdominalOperation[J].Chinese Journal of Urban and Rural Enterprise Hygiene , 2014(1): 59-60. 